\title{
Disagreement and Epistemic Utility-Based Compromise
}

\author{
Julia Staffel
}

Received: 30 October 2013 / Accepted: 6 May 2014

(C) Springer Science+Business Media Dordrecht 2014

\begin{abstract}
Epistemic utility theory seeks to establish epistemic norms by combining principles from decision theory and social choice theory with ways of determining the epistemic utility of agents' attitudes. Recently, Moss (Mind, 120(480), 1053-69, 2011) has applied this strategy to the problem of finding epistemic compromises between disagreeing agents. She shows that the norm "form compromises by maximizing average expected epistemic utility", when applied to agents who share the same proper epistemic utility function, yields the result that agents must form compromises by splitting the difference between their credence functions. However, this "split the difference" norm is in conflict with conditionalization, since applications of the two norms don't commute. A common response in the literature seems to be to abandon the procedure of splitting the difference in favor of compromise strategies that avoid non-commutativity. This would also entail abandoning Moss' norm. I explore whether a different response is feasible. If agents can use epistemic utilitybased considerations to agree on an order in which they will apply the two norms, they might be able to avoid diachronic incoherence. I show that this response can't save Moss' norm, because the agreements concerning the order of compromising and updating it generates are not stable over time, and hence cannot avoid diachronic incoherence. I also show that a variant of Moss' norm, which requires that the weights given to each agent's epistemic utility change in a way that ensures commutativity, cannot be justified on epistemological grounds.
\end{abstract}

Keywords Epistemic utility · Judgment aggregation · Disagreement · Conditionalization $\cdot$ Linear averaging $\cdot$ Scoring rule

J. Staffel (四)

Department of Philosophy, Washington University in St. Louis, One Brookings Drive,

St. Louis, MO 63130-4899 USA

e-mail: staffel.julia@gmail.com 


\section{Introduction}

Epistemic utility theory seeks to establish epistemic norms by combining decision theory and social choice theory with an account of the epistemic utility of agents' attitudes. Recently, Moss has applied this strategy to the problem of finding epistemic compromises between disagreeing agents [4]. She shows that the principle "form compromises by maximizing average expected epistemic utility", when applied to agents who share the same proper epistemic utility function, yields the result that agents must aggregate their judgments by splitting the difference between their credence functions. The principle employed by Moss is very similar to a familiar version of consequentialism, according to which one ought to perform the action that maximizes the expected total utility.

Unfortunately, the resulting "split the difference" norm is in conflict with a venerable norm of decision theory: updating by conditionalization. The problem is that the two norms don't commute: compromising first and then updating the compromise credence can yield a different joint credence than updating individual credences first and then compromising. A common response in the literature seems to be to abandon the procedure of splitting the difference in favor of compromise strategies that are externally Bayesian, i.e. that lead to the same joint credence independently of the order in which the agents compromise and update. This would also entail abandoning Moss' norm "maximize average expected epistemic utility." I explore whether a different response is feasible. If agents can use epistemic utility-based considerations to agree on an order in which they will apply the two norms, they might be able to avoid diachronic incoherence in their joint attitudes. I show that this response can't save Moss' norm, because the agreements concerning the order of compromising and updating it generates are not stable over time, and hence cannot avoid diachronic incoherence. I then explore whether changing Moss' norm in a way that enforces commutativity with conditionalization is plausible. I argue that the resulting norm is untenable, because it conflicts with some very plausible assumptions about how to determine the weight that is given to an expert's opinion in a compromise.

\section{The Situation}

Suppose that there are two agents, Alma and Berta, who disagree in their degrees of belief. They must somehow aggregate their conflicting attitudes, because they are asked to report their joint attitude on some proposition q tomorrow night. They know furthermore that they will find out tomorrow morning whether $\mathrm{p}$ is true or false, which will make a difference to their attitudes towards q. ${ }^{1}$

\footnotetext{
${ }^{1}$ The problem I am interested in here is how the agents should form a degree of belief that represents their joint opinion. The answer to this question does not automatically settle the answer to the question of whether and how the agents should change their individual credences when they learn about the disagreement.
} 
In order to generate the compromise attitude towards q, the agents must respond to learning whether $\mathrm{p}$ is the case, and they must find a compromise between their disagreeing degrees of belief, though not necessarily in this order. In the next section, I will present two norms that prescribe how these epistemic operations ought to be performed, and explain how epistemic utility theory is used to justify them.

\section{Epistemic Utility-Based Norms}

Given a suitable way of characterizing epistemic utility, we can use decision theory and social choice theory to defend epistemic norms. Such norms characterize epistemic states agents might adopt as rationally permissible or impermissible. For the purposes of our example two particular norms are relevant that have been supported by arguments of this kind: updating by conditionalization, and reaching epistemic compromises by splitting the difference between the agents' credences. I will briefly explain the arguments for each of these norms.

\subsection{Updating by Conditionalization}

The conditionalization norm requires all fully rational agents to update their credence functions in the following way:

Conditionalization One's new credence $\mathrm{Cr}_{\text {new }}(\mathrm{q})$ in a proposition $\mathrm{q}$ upon learning a proposition $\mathrm{p}$ should be one's old conditional credence $(\mathrm{q} \mid \mathrm{p})$, where $\operatorname{Cr}(\mathrm{q} \mid \mathrm{p})=$ $\mathrm{Cr}(\mathrm{p} \& \mathrm{q}) / \mathrm{Cr}(\mathrm{p})$, and $\mathrm{Cr}(\mathrm{p})>0$.

The conditionalization norm has been defended via a variety of arguments, but here I am only interested in the epistemic utility-based argument. It proceeds as follows: suppose an agent's epistemic values are represented by a proper scoring rule. In a nutshell, a scoring rule determines the epistemic value of an agent's credence in a proposition in a given world by measuring its distance from the truth-value of the proposition in that world. A scoring rule is proper if, according to it, any probabilistic credence function expects itself to have a higher epistemic utility than any alternative credence function an agent might adopt. One example of a proper scoring rule that is popular in the literature is the Brier score. ${ }^{2}$ If the agent's epistemic utility is measured by a proper scoring rule, it follows that updating by conditionalization is the only way of updating the agent's credences that maximizes her epistemic utility, relative to her pre-update credences and a partition from which the newly learned evidence will

\footnotetext{
${ }^{2}$ The Brier score is a proper scoring rule that can be used to measure the epistemic utility of an agent's credence, or credence function, at a given world. It is essentially a way of measuring how far away the agent's credences are from the truth - the larger the difference between an agent's credence in some proposition $\mathrm{p}$ and the truth value of $\mathrm{p}$ at that world, the higher the inaccuracy, or epistemic disutility, of that credence. More formally: suppose $c$ is a credence function defined over a set of propositions $F$, and the function $I$ indicates the truth values of the propositions in $F$ at world $w$ by mapping them onto $\{0,1\}$. Then the following function gives us the Brier score of $c$ at $w: \operatorname{Brier}(c, w)=\sum_{A \in F}(c(A)-I w(A))^{2}$
} 
come. In other words, if all the agent cares about is to maximize her expected epistemic utility, and her epistemic utility function can be captured by a proper scoring rule, then she is rationally required to update her credences via conditionalization $[3,5]$.

\subsection{Splitting the Difference}

The "split the difference" norm specifies how to aggregate the opinions of rational agents who disagree in their degrees of belief. It requires that agents reach a joint attitude by meeting in the middle between their individual attitudes.

Splitting the Difference For any finite group of rational agents $A_{1} \ldots A_{n}$, and any proposition $\mathrm{p}$, if $\mathrm{A}_{1} \ldots \mathrm{A}_{\mathrm{n}}$ 's credences in $\mathrm{p}$ are $\mathrm{x}_{1} \ldots \mathrm{x}_{\mathrm{n}}$, then their compromise credence in $\mathrm{p}$ must be $1 / \mathrm{n} \times\left(\mathrm{x}_{1}+\ldots+\mathrm{x}_{\mathrm{n}}\right)$.

Moss' epistemic utility-based argument for splitting the difference proceeds from the following norm: Agents who disagree about some proposition $\mathrm{p}$ should find a compromise by choosing the credence in $\mathrm{p}$ that maximizes their average expected epistemic utility [4]. ${ }^{3}$ This norm is equivalent to the norm that says that agents should maximize their joint expected epistemic utility. A similar norm in social choice theory is the following version of consequentialism: one ought to perform the action that maximizes the expected total utility. ${ }^{4}$

Moss argues that this norm is a plausible and intuitive way in which groups can find compromises that are best in light of their joint epistemic values. Furthermore, she points out that this norm is the epistemic analogue of a natural way of making joint practical decisions. She then proves that, given that the agents share the same proper scoring rule as their utility function, this compromising strategy prescribes that they must find their joint credence by splitting the difference between their individual credences.

Notice that this argument does not establish splitting the difference in its fully general form, since the argument requires that the agents must share the same utility function. Agents with differing epistemic utility functions may reach a different kind of compromise if they apply Moss' norm. However, we will assume henceforth that all agents we are talking about use the Brier score as their utility function, so splitting the difference applies to the cases we are considering.

\section{The Problem of Non-Commutativity}

Recall the example of Alma and Berta, who need to report their joint attitude in $\mathrm{q}$ tomorrow night, after they have found out whether $\mathrm{p}$ is true. Given the two norms

\footnotetext{
${ }^{3}$ To be precise, she says that it is not mandatory that both agents' attitudes get weighted equally, but doing so is the default strategy unless one of the agents has more expertise on the issue in question.

${ }^{4}$ As an anonymous referee helpfully points out, Moss' norm is not an exact analogue of consequentialism: standard consequentialism calculates which action has the highest expected utility based on a single agent's credences, whereas Moss' norm takes into account what each agent's expected epistemic utilities are, hence taking into account more than one agent's credences.
} 
introduced in the previous section, there are two natural ways in which Alma and Berta could find their compromise attitude towards q. Alma and Berta could compromise now by splitting the difference, and then update their compromise attitude about $\mathrm{q}$ by conditionalization when they find out about $\mathrm{p}$. Alternatively, Alma and Berta could each wait individually to find out whether $\mathrm{p}$ is true, update their individual attitudes, and subsequently form their compromise credence in q by splitting the difference.

If these procedures were known to yield the same result, then order wouldn't matter. However, it is well known that they don't commute: there are cases where compromising first and then updating the compromise credence yields a different result from updating individually first, and then compromising [2]. The following example illustrates how this can happen. The first table shows Alma's and Berta's initial credences, as well as the compromise credence function that is arrived at by splitting the difference between their unconditional credences.

\begin{tabular}{|l|l|l}
\hline Alma & Berta & Compromise \\
\hline $\mathrm{Cr}(\mathrm{p} \& \mathrm{q})=0.25$ & $\mathrm{Cr}(\mathrm{p} \& \mathrm{q})=0.6$ & $\mathrm{Cr}(\mathrm{p} \& \mathrm{q})=0.425$ \\
\hline $\mathrm{Cr}(\mathrm{p} \& \sim \mathrm{q})=0.35$ & $\mathrm{Cr}(\mathrm{p} \& \sim \mathrm{q})=0.2$ & $\mathrm{Cr}(\mathrm{p} \& \sim \mathrm{q})=0.275$ \\
\hline $\mathrm{Cr}(\sim \mathrm{p} \& \mathrm{q})=0.25$ & $\mathrm{Cr}(\sim \mathrm{p} \& \mathrm{q})=0.1$ & $\mathrm{Cr}(\sim \mathrm{p} \& \mathrm{q})=0.175$ \\
\hline $\mathrm{Cr}(\sim \mathrm{p} \& \sim \mathrm{q})=0.15$ & $\mathrm{Cr}(\sim \mathrm{p} \& \sim \mathrm{q})=0.1$ & $\mathrm{Cr}(\sim \mathrm{p} \& \sim \mathrm{q})=0.125$ \\
\hline $\mathrm{Hence}:$ & $\mathrm{Cr}(\mathrm{p})=0.8$ & $\mathrm{Cr}(\mathrm{p})=0.7$ \\
\hline $\mathrm{Cr}(\mathrm{p})=0.6$ & $\mathrm{Cr}(\sim \mathrm{p})=0.2$ & $\mathrm{Cr}(\sim \mathrm{p})=0.3$ \\
\hline $\mathrm{Cr}(\sim \mathrm{p})=0.4$ & $\mathrm{Cr}(\mathrm{q} \mid \mathrm{p})=0.75$ & $\mathrm{Cr}(\mathrm{q} \mid \mathrm{p})=0.607$ \\
\hline $\mathrm{Cr}(\mathrm{q} \mid \mathrm{p})=0.416$ & $\mathrm{Cr}(\mathrm{q} \mid \sim \mathrm{p})=0.5$ & $\mathrm{Cr}(\mathrm{q} \mid \sim \mathrm{p})=0.583$ \\
\hline $\mathrm{Cr}(\mathrm{q} \mid \sim \mathrm{p})=0.625$ & &
\end{tabular}

It's easy to see that the order in which Alma and Berta compromise and update makes a difference. First, let's look at the case where they learn that $p$ is true. If they compromise first, their joint credence in $(q \mid p)$ is 0.607 , so this will be their joint credence in $\mathrm{q}$ when they update their joint credence upon learning $\mathrm{p}$. By contrast, if they each learn $\mathrm{p}$ individually first, Alma will update her credence in $\mathrm{q}$ to 0.416 , and Berta hers to 0.75 . If they then split the difference, they arrive at a joint credence of 0.583 in q.

Next, let's see what happens in the case where they learn $\sim \mathrm{p}$. If they compromise first, their joint credence in $(\mathrm{q} \mid \sim \mathrm{p})$ is 0.583 , so this will be their joint credence in $\mathrm{q}$ when they update it on $\sim \mathrm{p}$. By contrast, if they each learn $\sim \mathrm{p}$ individually first, Alma will update her credence in $\mathrm{q}$ to 0.625 , and Berta hers to 0.5 . If they then split the difference, they arrive at a joint credence of 0.5625 in q. We see in each scenario that the resulting compromise credence in q depends on the order in which the agents update and compromise. How should we react to the result that splitting the difference and updating by conditionalization don't commute?

One reason to be worried about compromise norms that don't commute with conditionalization is that they can lead to diachronic incoherence in the compromise attitudes. For example, suppose that Alma and Berta form a joint credence in some 
proposition $\mathrm{p}$ by splitting the difference on Monday, then they individually learn new relevant information, and then they form a new compromise credence in $\mathrm{p}$ by splitting the difference on Tuesday. Since splitting the difference and conditionalization don't commute, there are cases in which their joint credence in $\mathrm{p}$ on Monday and their joint credence in $\mathrm{p}$ on Tuesday are not related in the way required by conditionalization. This is because the joint Tuesday credence is not the same credence we would have gotten by updating the joint Monday credence via conditionalization. And if this is the case, the agents' joint attitudes are diachronically incoherent, which makes them vulnerable to a diachronic Dutch book. ${ }^{5}$ If we reject splitting the difference for this reason, then we also have to reject Moss' norm "maximize average expected epistemic utility" as a norm that helps us find compromise strategies.

It is in fact a common reaction in the literature to argue that compromising strategies that don't commute with conditionalization are problematic. ${ }^{6}$ Splitting the difference is rejected in favor of some alternative compromise strategy. ${ }^{7}$ But maybe we don't need to reject splitting the difference, and hence Moss' norm, so quickly. It seems like Alma and Berta can avoid diachronic incoherence in their joint attitudes as long as they agree whether they will compromise first, and keep updating their compromise credence, or update first, and only compromise once all relevant information has been gathered. Of course, the latter proposal presupposes that the agents know how much evidence they will collect before they need to reach a compromise, and that they don't need to re-compromise later after learning additional information. This is an idealizing assumption, but as I will show, even under such favorable circumstances, Moss' norm runs into problems.

I will now explore whether agents can use the resources of epistemic utility theory to agree on a strategy for compromising and updating that avoids compromising multiple times, and hence avoids diachronic incoherence.

\section{Epistemic Utility Theory to the Rescue?}

We have seen that it makes a difference in what order Alma and Berta compromise and update. In order for them to avoid diachronic incoherence in their joint attitudes,

\footnotetext{
${ }^{5}$ See Russell, Hawthorne, \& Buchak, Groupthink, (manuscript), for an example of such a Dutch book. ${ }^{6}$ See, for example: Russell, Hawthorne \& Buchak, Groupthink, (manuscript); [1, 2, 7, 8], and others.

${ }^{7}$ This is of course not the only possible reaction to the data about non-commutativity. An alternative response would be for instance to reject conditionalization and/or to embrace diachronic incoherence. Usually, conditionalization is defined and defended as a norm that applies to a particular agent's credences. Hence, one might argue that the joint credences of groups are importantly different from the credences of a single agent, and conditionalization is a norm governing the latter, but not the former. However, it seems plausible to me that if a group wants to find a joint opinion in order to act as a single, unified epistemic agent, then the same norms apply to their joint opinion that govern an individual agent's credences. Hence, it seems difficult to reject conditionalization for joint opinions while accepting it for individual opinions. Moreover, given the variety and strength of the arguments for conditionalization, rejecting the rule altogether seems to be generally perceived as unattractive, and furthermore, it would be deeply revisionary.
} 
they must agree on a strategy and stick to it. How can they reach such an agreement? I propose that they should try to do so by using the resources of epistemic utility theory. The norms in question - splitting the difference and conditionalization - are supported by epistemic utility considerations. It seems very natural to propose that the same resources should be used by Alma and Berta to agree on a compromise strategy.

First I will investigate whether it is possible for Alma and Berta to agree on a compromise strategy based on individual epistemic utility considerations. In other words, if each of them determines which strategy (splitting the difference and then updating, or the reverse) maximizes their individual expected epistemic utility, can they reach an agreement? We will assume throughout that their credences are the ones given in section three, and that their epistemic utility function is captured by the Brier score. Let's walk through the example to see what happens.

Alma and Berta know that each of the potential final compromise credences in $\mathrm{q}$ will have a worse (=higher) expected Brier score for them than their final individual credences in q. ${ }^{8}$ If they choose a strategy based on individual expected utility considerations, they should each choose the strategy that they expect to lead to a lower expected Brier score increase, because, from each of their points of view, this is the strategy whose result is more accurate. ${ }^{9}$ Here's how Alma and Berta should calculate the results:

\begin{tabular}{|l|l|l|}
\hline & Choose compromising first & Choose updating first \\
\hline learn $\mathrm{p}$ & $\begin{array}{l}\text { increase in expected Brier score } \\
\text { compared to individual credence } \\
\text { in } \mathrm{q}: \mathrm{x}\end{array}$ & $\begin{array}{l}\text { increase in expected Brier score } \\
\text { compared to individual credence } \\
\text { in } \mathrm{q}: \mathrm{m}\end{array}$ \\
\hline learn $\sim \mathrm{p}$ & $\begin{array}{l}\text { increase in expected Brier score } \\
\text { compared to individual credence } \\
\text { in } \mathrm{q}: \mathrm{y}\end{array}$ & $\begin{array}{l}\text { increase in expected Brier score } \\
\text { compared to individual credence } \\
\text { in } \mathrm{q}: \mathrm{n}\end{array}$ \\
\hline
\end{tabular}

Calculate for each agent which strategy is expected to have a lower increase in expected Brier penalty for q:

\author{
Compromise first : $\mathrm{Cr}(\mathrm{p}) \mathrm{x}+\mathrm{Cr}(\sim \mathrm{p}) \mathrm{y}$ \\ Update first : $\mathrm{Cr}(\mathrm{p}) \mathrm{m}+\mathrm{Cr}(\sim \mathrm{p}) \mathrm{n}$
}

\footnotetext{
${ }^{8}$ This is because a coherent agent will always expect her own credences to have a lower Brier score than any alternative credences.

${ }^{9}$ Instead of using the increases in Brier score, we could also just work with the Brier scores for the different values of $q$. The result is exactly the same.
} 
Using this way of deciding on a strategy leads Alma to prefer updating first, and Berta to prefer compromising first. ${ }^{10}$ That is because Alma expects updating first to lead to a 0.0183 increase in the Brier score for $\mathrm{q}$, and she expects compromising first to lead to a 0.0226 increase in the Brier score for q. Berta expects updating first to lead to a 0.0231 increase in the Brier score for $\mathrm{q}$, and she expects compromising first to lead to a 0.0177 increase in the Brier score for q. Hence, they can't agree on a strategy that they both prefer by using individual expected epistemic utility calculations.

Yet, there is of course another alternative. Instead of using individual utility considerations, Alma and Berta can instead apply Moss' norm, and find out which strategy has a better average expected epistemic utility. In doing so, we're expanding the range of application of Moss' norm. It was originally intended to be used

${ }^{10}$ Let's walk through the calculations for Alma, to make things more concrete (numbers are rounded):

If Alma learns that $p$, her new credence in $q$ will be 0.416 . She will then expect the following Brier score for her credence in q:

$$
0.416(0.416-1)^{2}+0.584(0.416-0)^{2}=0.2429
$$

She can compare this to the Brier scores she expects for the compromise credences the two strategies yield for $\mathrm{q}$.

Compromise first:

$$
0.416(0.607-1)^{2}+0.584(0.607-0)^{2}=0.2794
$$

Expected Brier penalty increase: $0.0365(=\mathrm{x})$

Update first:

$$
0.416(0.583-1)^{2}+0.584(0.583-0)^{2}=0.2708
$$

Expected Brier penalty increase: $0.0279(=\mathrm{m})$

If Alma learns that $\sim p$, her new credence in $q$ will be 0.625 . She will then expect the following Brier score for her credence in q:

$$
0.625(0.625-1)^{2}+0.375(0.625-0)^{2}=0.2344
$$

She can compare this to the Brier scores she expects for the compromise credences the two strategies yield for $\mathrm{q}$.

Compromise first:

$$
0.625(0.583-1)^{2}+0.375(0.583-0)^{2}=0.2361
$$

Expected Brier penalty increase: $0.00176(=y)$

Update first:

$$
0.625(0.563-1)^{2}+0.375(0.563-0)^{2}=0.2383
$$

Expected Brier penalty increase: $0.00391(=\mathrm{n})$

Now, Alma can calculate which strategy she expects to have a lower increase in expected Brier penalty:

$$
\begin{aligned}
\text { Compromise first : } & \operatorname{Cr}(\mathrm{p}) \mathrm{x}+\mathrm{Cr}(\sim \mathrm{p}) \mathrm{y} \\
& 0.6 \times 0.0365+0.4 \times 0.00176=0.0226 \\
\text { Update first : } & \mathrm{Cr}(\mathrm{p}) \mathrm{m}+\mathrm{Cr}(\sim \mathrm{p}) \mathrm{n} \\
& 0.6 \times 0.0279+0.4 \times 0.00391=0.0183
\end{aligned}
$$

As we can see, Alma expects updating first to have a lower increase in expected Brier score, so this is the strategy she prefers based on individual expected epistemic utility calculations. The calculations for Berta proceed in the same fashion, and show that she prefers compromising first:

$\begin{array}{ll}\text { Compromise first : } & 0.8 \times 0.02045+0.2 \times 0.00689=0.01774 \\ \text { Update first: } & 0.8 \times 0.02789+0.2 \times 0.00391=0.02309\end{array}$ 
for finding a compromise between agents' current credences, by determining which joint credence would maximize average expected epistemic utility. I suggest that we can also use it to choose a compromising strategy, i.e. an order of updating and compromising that eventually leads to a joint credence.

We previously used expected epistemic utility calculations to see which strategy would be preferred by Alma and Berta individually, and we saw that they didn't prefer the same strategy. We can use the results from these calculations in order to determine which strategy they jointly prefer. We simply compare the sums of the expected Brier scores of the two strategies, and see which one fares better. (The result is the same if we take the average of these sums.) We already have the results we need in order to do this (see the end of footnote 10):

\section{Compromise first $(\mathrm{CF})$ :}

Expected Brier score increase $(\mathrm{CF}, \mathrm{Alma})+$ Expected Brier score increase $(\mathrm{CF}, \mathrm{Berta})$ $0.0226+0.0177=0.0403$

\section{Update first (UF):}

Expected Brier score increase (UF, Alma) + Expected Brier score increase (UF, Berta) $0.0183+0.0231=0.0414$

This way of deciding about the order of compromising and updating leads Alma and Berta to decide that they should compromise first. This is because the sum of the expected Brier score increases is slightly lower for compromising first than for updating first. If Alma and Berta decided to form their final joint credence in $\mathrm{q}$ in this way, by splitting the difference between their initial credences, and then updating their compromise credence in $q$ upon learning whether $p$, they could avoid having diachronically incoherent joint credences.

Unfortunately, there's still a problem: suppose Alma and Berta settle on the strategy that involves compromising first. They compromise, and then they find out whether $\mathrm{p}$ is true. They update their joint credence accordingly, as well as their individual credences. Yet, once they've done so, they will notice that Moss' norm no longer recommends that they report the credence in q produced by the compromise-first strategy as their joint credence. It is a direct consequence of Moss' result that once Alma and Berta have learned whether $\mathrm{p}$ is true individually, the norm "maximize average expected utility" will tell them that averaging their current, updated credences is optimal. But if they decide to compromise between their updated credences, their credences are again diachronically incoherent, because the joint credence they end up adopting in $q$ is different from the credence in $q$ they anticipated to adopt.

What this example shows is that we cannot save Moss' norm by making agents use it to choose updating strategies. If it had turned out that agents always preferred strategies in which they compromise last, after they have individually learned the available information, then there would be no diachronic incoherence - they would prefer compromising last both before and after learning relevant information. However, our example shows that there are cases in which Moss' norm recommends compromising before updating, but this recommendation changes once the agents 
have updated their individual credences, leading to diachronically incoherent joint credences.

One might try to respond by emphasizing that the whole point of picking a strategy is that the agents must stick to it, i.e. they don't get to reconsider what they want their joint credence to be after they learn new information. However, I don't think this response succeeds. Binding oneself to a strategy is usually considered a good idea if one is initially in a better position to assess what the best strategy is than after one has made further decisions. For example, an agent might decide before a party to have only one drink and then go home. She shouldn't reconsider this strategy after she's already had one drink, because in her slightly tipsy state she will prefer to continue drinking, leading to consequences she wants to avoid. However, our example doesn't work this way. Alma and Berta's position to decide on a compromise credence after they've learned about $\mathrm{p}$ is at least as good, if not better, than before they learn about p. Hence, it's not plausible that they should be banned from reconsidering their initial strategy after they've found out about $\mathrm{p}$.

Moreover, while reconsidering in our example leads to diachronic incoherence, this feature doesn't generalize to all cases, since what agents prefer depends on their particular credence functions. There are cases in which reconsidering is not a problem. In cases where the agents preferred compromising last all along, or where they were indifferent between different compromising strategies, it would be fine for them to reconsider their compromise strategy by applying Moss' norm to their most recent credences, because they would just agree that their earlier decision was right, and that they still endorse the compromise credence that is prescribed by the strategy they agreed upon earlier.

Hence, there doesn't appear to be any non-ad hoc reason to systematically ban agents from reapplying Moss' norm to their current credences. Doing so neither has bad consequences in every case, nor have the agents become epistemically deficient in ways that would warrant banning them from reapplying the norm. To the contrary, their epistemic situation is arguably better than when they first agreed on a strategy.

\section{The Changing Weights Strategy}

I argued in the previous section that we cannot rescue Moss' norm as it stands by getting the agents to agree on a compromising strategy that avoids diachronic incoherence. In this section, I will explore whether a slightly different version of Moss' norm that avoids non-commutativity fares better.

It has long been known that the commutativity problem arises for any compromise norm that requires forming a weighted linear average between the agents' credences, and that keeps the weight given to each individual agent constant. In our example, the splitting the difference norm requires that we give equal weight to Alma's and Berta's credences before and after they learn whether $\mathrm{p}$ is true, hence the weight given to their individual credences remains constant. However, commutativity can be achieved if the post-update weights for aggregating the agents' credences 
are computed from the pre-update weights via a particular formula. Generally, if $\mathrm{w}_{\mathrm{i}}$ is the weight assigned to $\mathrm{Cr}_{\mathrm{i}(\mathrm{old})}(\mathrm{q})$ (the agent $\mathrm{i}$ 's pre-update credence in $\mathrm{q}$ ), and $v_{i}$ is the weight assigned to $\mathrm{Cr}_{i(\mathrm{old})}(\mathrm{q} \mid \mathrm{p})$, which equals $\mathrm{Cr}_{\mathrm{i}(\mathrm{new})}(\mathrm{q})$, then $\mathrm{w}_{\mathrm{i}}$ and $\mathrm{v}_{\mathrm{i}}$ must be related as follows:

$$
v_{i}=\frac{w_{i} C r_{i(\text { old })}(p)}{\sum_{j}^{n} w_{j} C r_{j(o l d)}(p)}
$$

The denominator is the weighted sum of all the probability assignments to $\mathrm{p}$ we want to aggregate $[6,7]$. Hence, what determines the post-update weights given to agents' credences are (i) the pre-update weights, and (ii), the agents' pre-update credences in the proposition that is learned. If the weights given to each agent's credences are adjusted in this way each time the agents in a group learn new information, then their pre- and post-update compromise credences are related in the way required by conditionalization.

In our example, this yields the following results if we assume that the initial weights for Alma and Berta's credences are 1/2:

New weight for Alma's credence in $\mathrm{q}: 0.5 \times 0.6 / 0.5 \times 0.6+0.5 \times 0.8=0.4286$

New weight for Berta's credence in $q: 0.5 \times 0.8 / 0.5 \times 0.6+0.5 \times 0.8=0.5714$

If we use these weights for aggregating Alma's and Berta's credences after they individually learn that $\mathrm{p}$, the result will be the same as if we update their joint credence on p.

Now we know how to adjust the "split the difference" norm in order to avoid noncommutativity. How does this carry over to Moss' norm "maximize average expected epistemic utility"? As Moss shows, for agents who use the same proper scoring rule, the weight given to each agent's expected epistemic utility is the same as the weight given to her credence when aggregating [4]. Hence, Moss could endorse a version of her norm according to which the weight given to each agent's expected epistemic utility is determined by the formula above (at least in cases where they share the same proper scoring rule). For example, if two agents start out using Moss' original norm, which gives each of their expected epistemic utilities a weight of $1 / 2$, then, whenever they learn something, the weights given to their expected epistemic utilities in a post-update compromise must be adjusted as described above. I will henceforth refer to this proposal as the 'changing weights-version' of Moss' norm. Adopting it would help us avoid the commutativity problem. In principle, it seems like Moss might be amenable to such a suggestion. While she thinks that maximizing the average expected epistemic utility of the disagreeing agents is suitable in cases in which agents of equal expertise seek a fair compromise, she points out there can be other cases in which there are good reasons not to give each agent equal weight, for example if one agent is an expert and the other agent is a layperson.

We have established that the changing weights-version of Moss's norm commutes with conditionalization. In order to determine whether we should accept it, we need to examine whether the weights prescribed by the norm seem reasonable given some natural claims about how a person's epistemic situation is related to how much weight we should give their opinions. For example, consider two agents who are both experts 
in some particular field, such as weather forecasting or forensic science. Each agent has worked in the field for several decades, and their equally excellent track records indicate their expertise in their field. It seems quite natural to think that, should they disagree on some issue in their field of expertise, the two agents' opinions should be given equal weight. Moreover, it seems quite plausible that a few inaccurate predictions on the part of one of the agents should not be sufficient to question her expertise. One of the agents would have to have a somewhat sustained run of bad judgments in order to be demoted from her status as an expert.

Unfortunately, the weights required by the changing weights-version of Moss' norm are much more shifty than they intuitively should be. How drastically the weights can shift can be illustrated with an example. Suppose Alma's and Berta's opinions are initially given equal weight, i.e 0.5 . They come to learn some proposition p, which Alma initially believes to degree 0.2, and Berta believes to degree 0.8. This is all that's needed to calculate the post-update weights for Alma's and Berta's credences, and it is easy to determine that Alma's credences now get a weight of 0.2 , and Berta's credences get a weight of 0.8 . In other words, based on the fact that Berta's credence was closer to the truth-value of the learned proposition in a single instance, her opinion now gets four (!) times as much weight as Alma's opinion in the post-update compromise.

We saw that the post-update weights given to agents' credences are calculated on the basis of the pre-update weights, and the agents' pre-update credences in the proposition that is learned. The long-term track record that we usually take to determine an agent's expertise enters the equation in the form of the pre-update weight, but as the example illustrated, the pre-update weight can differ dramatically from the post-update weight, depending on what credences each agent assigned to p. An agent whose credence in $\mathrm{p}$ was closer to p's truth-value will get assigned comparatively more weight than an agent whose credence in $\mathrm{p}$ was farther away from p's truth-value. But surely, how well each agent estimated the truth-value of a single proposition should not justify such drastic shifts in how we weigh their opinions!

Notice that it doesn't even matter what the content of $p$ is. It might be that the content of $\mathrm{p}$ is only marginally relevant to the subject matter on which the agents seek to form a compromise, which means that how accurate the agents' estimate of p's truth-value is tells us hardly anything about their expertise on the subject matter at hand. Hence, their accuracy with respect to $\mathrm{p}$ should hardly move us to change how much weight we give to each expert's opinion. ${ }^{11}$ Moreover, the agent whose credence in $\mathrm{p}$ was closer to p's truth-value did not thereby automatically have a more justified opinion. Sometimes, unlikely things happen, and if p's objective chance was known to be low, then the agent who assigned $\mathrm{p}$ a low credence had a more justified opinion than the agent whose credence in $\mathrm{p}$ was high.

Hence, these considerations show quite clearly that the shifts in weight required to ensure commutativity are not the kinds of shifts that seem reasonable if we think that an agent's expertise, based on her long-term track record, determines how much

\footnotetext{
${ }^{11}$ A similar point was made by Leon Leontyev in a talk entitled "Conciliating with Conditionalisation", given at the Australian National University in September 2013.
} 
weight her opinion should be given in a compromise. Moreover, it is hard to see what other epistemically relevant factors besides an agent's expertise could be invoked to explain that the weights given to each agent's opinion should always change exactly as the formula prescribes.

One could surely imagine that agents might adopt the kind of compromising strategy prescribed by the changing weights-version of Moss' norm for practical reasons, for example because they find it convenient to employ, or because they cannot agree on a better solution. However, Moss' original intention, and the general motivation behind epistemic utility theory, was to motivate epistemic norms based on epistemological, rather than pragmatic considerations. Hence, I conclude that the changing weights-version of Moss' norm cannot be motivated on epistemological grounds, even though it has the advantage of avoiding the commutativity problem. ${ }^{12}$

\section{Conclusion}

Moss' aim was to provide a compromise norm within the framework of epistemic utility theory that compliments the existing norms for individual agents. According to her, "the scoring rules strategy for compromising is a natural way of extending accepted norms governing single agents to norms governing compromise." Moreover, her proposal seemed initially like an attractive epistemic analogue of a familiar version of consequentialism.

I have argued that Moss' proposal is unsuccessful, and that it can't be defended even with adjustments. I first pointed out the well-known fact that the "split the difference" norm, which is prescribed by her norm "maximize average expected epistemic utility" when agents share the same proper scoring rule, doesn't commute with conditionalization. Some authors recommend abandoning splitting the difference for this reason. This would also entail abandoning Moss' norm.

I then explored whether Moss' norm could be saved by applying it to compromising strategies. If agents could agree on the order in which they update their credences and form compromises, they could potentially avoid diachronic incoherence between their joint credences. I showed that agents can't always agree on such a strategy based on individual utility considerations. If we instead used Moss' norm "maximize average expected utility" to find an order in which the agents update and compromise, we saw that no stable compromise could be reached in our example. The joint credence resulting from the strategy they prefer before learning about $\mathrm{p}$ no longer seems optimal to them after they learn about $\mathrm{p}$.

\footnotetext{
${ }^{12}$ An anonymous referee is more optimistic than I am about the changing weights-version of Moss' norm. The referee suggests that in light of how difficult it is to find an appropriate compromising strategy, this proposal might be among the more promising available options. S/he suggests that if we individuate areas of expertise very narrowly, the quick shifts in weight that can happen on this view might be justifiable. While I agree that finding a promising compromise rule is difficult, I still think it is a problem that the changing weights-version of Moss' norm is only sensitive to how close an agent's credence was to the truth value of the proposition in question, but not to how justified the agent's credence was.
} 
I then investigated whether the changing weights-version of Moss' norm is a better alternative. This version of the norm requires that the weights given to each agent's expected epistemic utility shift according to a formula that ensures that the resulting compromises commute with conditionalization. While this is an advantageous formal feature, I argued that the shifts in weight required by this norm can't be justified from an epistemological point of view.

Acknowledgments A substantial part of this paper was written while I was a postdoctoral fellow at the Australian National University, thanks to the Australian Research Council Grant for the Discovery Project 'The Objects of Probabilities', DP 1097075. For helpful comments and discussion of the paper, I would like to thank Jacob Ross, Kenny Easwaran, Brian Talbot, Dom Bailey, Leon Leontyev, Daniel Nolan, Paul Bartha, Yoaav Isaacs, Sharon Berry, and the audiences at the Central APA Meeting in 2013, the AAP Meeting in 2013, and the University of Sydney.

\section{References}

1. Fitelson, B., \& Jehle, D. (2009). What is the "equal weight view"? Episteme, 6(3), 280-293.

2. Genest, C., \& Zidek, J. (1986). Combining probability distributions: a critique and annotated bibliography. Statistical Science, 1(1), 114-135.

3. Greaves, H., \& Wallace, D. (2006). Justifying conditionalization: conditionalization maximizes expected epistemic utility. Mind, 115(459), 607-632.

4. Moss, S. (2011). Scoring rules and epistemic compromise. Mind, 120(480), 1053-69.

5. Pettigrew, R. (2013). Epistemic utility and norms for credences. forthcoming in Philosophy Compass.

6. Raiffa, H. (1968). In Decision analysis. Reading: Addison-Wesley.

7. Wagner, C. (1985). On the formal properties of weighted averaging as a method of aggregation. Synthese, 62(1), 97-108.

8. Wilson, A. (2010). Disagreement, equal weight, and commutativity. Philosophical Studies, 149(3), 321-326. 